\title{
Pharmacokinetics of a Novel Sildenafil Orodispersible Film Administered by the Supralingual and the Sublingual Route to Healthy Men
}

\author{
Luca Loprete $^{1} \mathbb{D} \cdot$ Chiara Leuratti $^{1} \cdot$ Valeria Frangione $^{2} \cdot$ Milko Radicioni $^{1}$
}

Published online: 16 June 2018

(c) The Author(s) 2018

\begin{abstract}
Background Sildenafil was the first selective drug available on the market as oral therapy for erectile dysfunction (ED). A novel sildenafil orodispersible film (ODF) for ED treatment, containing sildenafil citrate, has recently been marketed.

Objectives Study objective was to investigate sildenafil bioavailability of the novel ODF formulation after sublingual and supralingual administration.

Methods In this randomised, two-way cross-over study, 12 healthy male volunteers received a single $50 \mathrm{mg}$ sildenafil dose by the sublingual and supralingual administration routes. Plasma sildenafil was determined up to $12 \mathrm{~h}$ post-dose. Peak concentration $\left(C_{\max }\right)$ and area under concentration-time curve $\left(\mathrm{AUC}_{0-t}\right)$ were calculated and compared between the two administration routes by analysis of variance (ANOVA).

Results Sublingual and supralingual administration can be claimed equivalent regarding the extent of sildenafil exposure since $\mathrm{AUC}_{0-t} 90 \%$ CIs corresponded to $94.90-110.58 \%$ and were within the pre-specified acceptance range. $C_{\max } 90 \%$ CIs (79.92-125.57\%) were only slightly outside the $80.00-125.00 \%$ limits, due to the small sample size, while the time to achieve $C_{\text {max }}$ did not differ between treatments $(p=0.9277)$. Rate of exposure of the two administration routes was therefore similar. Reported treatment-related adverse events were mild to moderate headache (33.3\% of subjects) and vomiting (8.3\%).

Conclusions In healthy men, sublingual and supralingual administration of sildenafil ODF resulted in a remarkably similar pharmacokinetic profile and confirmed the safety of both study treatments. The recently marketed sildenafil ODF, administered by both investigated routes, can provide a valuable alternative to the marketed solid oral forms (tablets) in ED treatment.
\end{abstract}

\section{Key Points}

In healthy men, sublingual and supralingual administrations of sildenafil orodispersible film resulted in a remarkably similar pharmacokinetic profile and confirmed the safety of both study treatments.

The recently marketed orodispersible film, administered by both investigated routes, can provide a valuable alternative to the marketed solid oral forms (tablets) in erectile dysfunction treatment.

Luca Loprete

luca.loprete@croalliance.com

1 CROSS Research S.A., Via F.A. Giorgioli 14, 6864 Arzo, Switzerland

2 IBSA Institut Biochimque S.A., Via del Piano, 6915 Pambio-Noranco, Lugano, Switzerland

\section{Introduction}

Penile erection is the result of smooth muscle relaxation in the penis. It includes arterial dilatation, trabecular smooth muscle relaxation, and activation of the corporeal venoocclusive mechanism [1-3]. Erectile dysfunction (ED) is defined as the persistent inability to attain and maintain a penile erection sufficient to permit satisfactory sexual performance [1-3]. Phosphodiesterase type 5 (PDE5) is a regulator of vascular smooth muscle contraction in all smooth muscle districts and especially in penis, and PDE5 inhibitors are currently the first-line therapy for ED.

Sildenafil was the first selective inhibitor of cGMP-specific PDE5 available on the market as oral therapy for ED [2-6].

Sildenafil is rapidly absorbed, with maximum observed plasma concentrations $\left(C_{\max }\right)$ reached within 30-120 min (median time $60 \mathrm{~min}$ ) after oral administration under fasting conditions. The mean absolute oral bioavailability is $41 \%$ 
(range 25-63\%). The area under the concentration-time curve (AUC) and $C_{\max }$ increase proportionally with the dose over the recommended oral dose range $(25-100 \mathrm{mg}$ ) indicating a dose-proportional rate and extent of absorption. When sildenafil is taken after a heavy and fatty meal, the rate of absorption is reduced with a delay in $t_{\max }$ and a mean reduction in $C_{\max }$ by $29 \%[7,8]$. The mean steady-state sildenafil volume of distribution is $105 \mathrm{~L}$, indicating distribution into the tissues. The total sildenafil body clearance is $41 \mathrm{~L} / \mathrm{h}$ with a resultant terminal half-life $\left(t_{1 / 2}\right)$ of $3-5 \mathrm{~h}$. Sildenafil is cleared predominantly by the CYP3A4 (major route) and CYP2C9 (minor route) hepatic microsomal isoenzymes. The major circulating metabolite, resulting from $\mathrm{N}$-demethylation of sildenafil, has a PDE selectivity profile similar to sildenafil and an in vitro potency for PDE5 approximately $50 \%$ of the parent drug. Plasma concentrations of this metabolite are approximately $40 \%$ of those found for sildenafil. The $N$-desmethyl metabolite is further metabolised, with a $t_{1 / 2}$ of approximately $4 \mathrm{~h}[9,10]$. The drug and its major circulating $N$-desmethyl metabolite are bound to plasma proteins in the amount of $96 \%$ and binding is independent of total drug concentrations [7, 11].

The good tolerability and safety profile of sildenafil for treating ED was established in approximately 74 doubleblind placebo-controlled trials performed in more than 9000 patients, confirming that sildenafil is well tolerated at the recommended dose regimen. The most commonly reported adverse reactions in clinical studies among sildenafil-treated patients were headache, flushing, dyspepsia, nasal congestion, dizziness, nausea, hot flush, visual disturbance, cyanopsia and blurred vision. Adverse reactions from post-marketing surveillance have been gathered covering an estimated period $>10$ years [12].

A novel sildenafil orodispersible film (ODF) containing sildenafil citrate has recently been marketed. The ODF formulation disintegrates rapidly in the oral cavity, usually within few minutes, without drinking or chewing, thus providing a valuable alternative to the marketed solid oral forms (tablets) in ED treatment. The ODF, available in 4 different dosage forms (i.e. $25,50,75$ and $100 \mathrm{mg}$ ), obtained marketing authorisation in Switzerland and several European Countries in 2016 and has been very recently made available on the market.

In a previous single-dose, randomised, two-way crossover Phase I study [13], the bioequivalence between the new Sildenafil IBSA $100 \mathrm{mg}$ ODF, administered supralingually, and the marketed Viagra ${ }^{\circledR}$ 100-mg film-coated tablet, Pfizer Limited UK, was proven in terms of rate and extent of sildenafil absorption after single-dose administration [14]. In addition, no significant differences in sildenafil and $\mathrm{N}$-desmethyl-sildenafil $t_{\max }$ values between the two products were observed, confirming that no significant sildenafil absorption differences occurred in the oral cavity after supralingual administration. However, due to its higher vascularisation with respect to the rest of oral cavity, the sublingual mucosa represents a specific route of absorption, that can possibly lead to modifications in the rate, extent or subject variability of drug absorption [15]. For this reason, in the present pilot study, the possibility of significant differences in sildenafil bioavailability after sublingual and supralingual administration of sildenafil ODF formulation was investigated. The pharmacokinetics of sildenafil $\mathrm{N}$-desmethyl metabolite and the safety profile of the study treatments were also investigated.

\section{Methods}

\subsection{Study Design and Procedures}

The study protocol (code 17CH-SDF06) was approved by the Ethics Committee of Canton Ticino, Switzerland, and the Swiss Federal Health Authorities. All subjects were given a detailed description of the study and all gave written informed consent before enrolment. The study was performed in accordance with the Declaration of Helsinki and harmonised European standards for Good Clinical Practice (ICH E6 1.24), from November to December 2017.

The study was single-centre, single-dose, open, randomised, two-way cross-over and was designed according to the EMA guideline for bioequivalence studies [14].

The investigational product was sildenafil ODF, a thin, flexible, opaque, light blue, orodispersible film (IBSA Institut Biochimique S.A., Switzerland) containing 70.2 $\mathrm{mg}$ of sildenafil citrate, equivalent to $50 \mathrm{mg}$ of sildenafil. It was orally administered in two study periods, below the tongue (tested alternative method of administration) and above the tongue (the approved method of administration of the product), with a wash-out interval of 6 days between administrations.

The study randomisation list was computer-generated by the Biometry Unit of CROSS Research S.A., Switzerland, using the PLAN procedure of the SAS/STAT ${ }^{\circledR}$ software version 9.3.

Subjects were confined at the clinical centre from the evening before administration up to $12 \mathrm{~h}$ post-dose and were in fasting conditions for $10 \mathrm{~h}$ before administration and up to $4 \mathrm{~h}$ post-dose. Water was allowed as desired, except for $1 \mathrm{~h}$ before and $1 \mathrm{~h}$ after dosing. In both study periods, the investigational product was administered on day 1 at $08: 00 \pm 1 \mathrm{~h}$ and under fasting conditions. The subjects took a standardised, small amount of still mineral water $(20 \mathrm{~mL})$ to moisten the oral cavity just before the ODF intake.

Thereafter, the investigator placed the investigational product either beneath the volunteer's tongue after folding the ODF once (sublingual route) or directly on the 
volunteer's tongue, taking care not to fold the ODF (supralingual route). Once administered, after the film had dissolved completely (without chewing), the subjects could swallow saliva.

\subsection{Subjects}

Healthy male volunteers aged 18-45 years, with a body mass index of $18.5-30.0 \mathrm{~kg} / \mathrm{m}^{2}$, were enrolled in the study. All volunteers were in good physical health, as assessed through full physical examination, electrocardiogram (ECG) recording, vital signs measurement and clinical laboratory assays, according to the study inclusion criteria. No subjects were on abnormal diets or had a history of drug, alcohol, caffeine or tobacco abuse. Exclusion criteria included history or presence of significant diseases, history of vision or hearing problems related to drugs of the PDE5 inhibitor pharmacological class, history of priapism; anatomical deformation of the penis, history of ophthalmologic diseases and hypersensitivity or allergic reactions to sildenafil. Medications, including organic nitrates, were not allowed for 4 weeks before the study, while over-the-counter drugs and herbal remedies were not allowed for 2 weeks before screening. Subjects were not enrolled if they had participated in other clinical trials or donated blood in the past 3 months.

\subsection{Blood Sampling}

Blood samples for sildenafil and $N$-desmethyl-sildenafil measurements were collected at pre-dose $(0)$ and $6,15,30$, 45 min and 1, 1.25, 1.5, 2, 2.5, 3, 4, 6, 8, 12 h post-dose. Sampling time points were selected based on previously reported data [13].

Blood samples were collected using an indwelling catheter with switch valve. The cannula was rinsed, after each sampling, with about $1 \mathrm{~mL}$ of sterile saline solution containing $20 \mathrm{IU} / \mathrm{mL}$ Na-heparin. The first $2 \mathrm{~mL}$ of blood were discarded at each collection time to avoid contamination of the sample with heparin. The remaining $8 \mathrm{~mL}$ were collected from the catheter and transferred with a syringe into heparinised tubes (Li-heparin). The samples were stored on ice for a maximum of $20 \mathrm{~min}$ and then centrifuged at $4{ }^{\circ} \mathrm{C}$ for $10 \mathrm{~min}$ at $2500 \mathrm{~g}$ to obtain plasma. Each plasma sample was immediately transferred into pre-labelled polypropylene tubes, and stored frozen at $\leq-20{ }^{\circ} \mathrm{C}$.

\subsection{Bioanalytical Assay}

Concentrations of sildenafil and $\mathrm{N}$-desmethyl sildenafil in plasma were determined by a blinded analyst at Analytisch Biochemisch Laboratorium BV (ABL), the Netherlands, using a LC-MS/MS method developed and validated according to the requirements of the EMA and
FDA guidelines on bioanalytical method validation [16, 17]. The method had a lower quantification limit (LQL) of $0.5 \mathrm{ng} / \mathrm{mL}$ and an upper quantification limit (UQL) of $2000 \mathrm{ng} / \mathrm{mL}$ for both analytes and adhered to the regulatory requirements for selectivity, sensitivity, precision, accuracy, recovery, carry-over, matrix effect, and stability.

Internal standards for the analysis were deuterated forms of the analytes (sildenafil- $\mathrm{D}_{8}$ and $\mathrm{N}$-desmethyl sildenafil- $\mathrm{D}_{8}$ ).

Calibration standards for sildenafil and for $\mathrm{N}$-desmethyl sildenafil in the range from 0.500 to $2000 \mathrm{ng} / \mathrm{mL}$ were prepared freshly in human Li-heparin plasma on the day of analysis.

Sildenafil and $N$-desmethyl sildenafil QC samples at the levels low $(1.50 \mathrm{ng} / \mathrm{mL})$, medium $1(75.0 \mathrm{ng} / \mathrm{mL})$, QC medium $2(1000 \mathrm{ng} / \mathrm{mL})$ and high $(1600 \mathrm{ng} / \mathrm{mL})$ were prepared in a batch and stored at $\leq-18^{\circ} \mathrm{C}$ prior to the start of the bioanalytical study.

Isolation of sildenafil, $N$-desmethyl sildenafil, sildenafil$\mathrm{D}_{8}$ and $N$-desmethyl sildenafil- $\mathrm{D}_{8}$ from human Li-heparin plasma was performed by liquid/liquid extraction (LLE) with methyl tert-butyl ether. After extraction, the organic layer was evaporated to dryness under a gentle stream of nitrogen. The residue was reconstituted in injection solvent. Chromatographic separation was performed on a Waters XTerra MS C18 column using gradient elution. An API 4000 tandem mass spectrometry equipped with a turbo spray ionization source operating in the positive multiple reaction monitoring mode was used for quantification.

Data acquisition was performed using Analyst software (version 1.6.2) from AB Sciex. Following peak area integration, regression was also performed using Analyst. Concentrations were calculated using 13-point curves with weighted linear regression.

\subsection{Pharmacokinetic Parameters}

Pharmacokinetic parameters were determined or calculated using the validated software Phoenix WinNonLin ${ }^{\circledR} 6.3$ (Certara, Inc). The primary study outcome measures were plasma sildenafil peak concentration $\left(C_{\max }\right)$, time to $C_{\max }$ $\left(t_{\max }\right)$ and area under the concentration-time curve up to the last sampling point $\left(\mathrm{AUC}_{0-t}\right)$ and extrapolated to infinity $\left(\mathrm{AUC}_{0-\infty}\right)$, calculated using the linear trapezoidal rule. The primary end-point of the study was the evaluation of the similarity of the two administration routes in terms of rate $\left(C_{\max }\right.$ and $\left.t_{\max }\right)$ and extent $\left(\mathrm{AUC}_{0-t}\right)$ of plasma sildenafil concentration. The following sildenafil pharmacokinetic parameters were also calculated: terminal volume of distribution $\left(V_{z} / F\right)$, total clearance $(\mathrm{Cl} / F)$ and half-life $\left(t_{1 / 2}\right)$. The same pharmacokinetic parameters were also calculated for the metabolite $N$-desmethyl-sildenafil, except for $V_{z} / F$ and $\mathrm{Cl} / F$. 


\subsection{Safety}

The safety profile of the investigational product was assessed by evaluating treatment-emergent adverse events (AEs), physical examination, ECG, routine laboratory tests and vital signs checks. Vital signs (blood pressure and heart rate) were measured at screening, final visit, and on day 1 of each study period at pre-dose, 1.75 , and $5 \mathrm{~h}$ post-dose. A 12-lead resting ECG was recorded at screening and final visit. Blood and urine samples were collected for routine haematology, blood chemistry, virology and urinalysis at screening and final visit. AEs were assessed throughout the study and were coded using MedDRA ${ }^{\circledR}$ version 20.1. A full physical examination was performed by the investigator at screening and at the final visit.

\subsection{Sample Size and Statistical Analyses}

Twelve healthy men were included in the study. The sample size was not based on any statistical evaluation, considering the exploratory purpose of the study.

The statistical analyses were performed using SAS $^{\circledR}$ software version 9.3 (TS1M1) for Windows ${ }^{\circledR}$ and Phoenix WinNonLin ${ }^{\circledR} 6.3$, Certara Inc.

A classical bioequivalence test was used for the comparison of sildenafil and $\mathrm{N}$-desmethyl-sildenafil pharmacokinetic parameters [14]. Log-transformed $C_{\max }, \mathrm{AUC}_{0-t}$ and $\mathrm{AUC}_{0-\infty}$ were analysed by ANOVA, with treatment, period, sequence and subject-within-sequence as fixed effects. Similarity criterion was a geometric means ratio (PE) around $100 \%$ and its 90 $\%$ confidence interval (CI) within the range $80.00-125.00 \%$. Wilcoxon signed-rank test was used to analyse $t_{\max }$.

\section{Results}

\subsection{Subjects}

Nineteen subjects were screened and twelve were randomised in the study. They received the treatment by the allocated route of administration, completed the study per protocol and were included in the pharmacokinetic and safety analyses. Demographic characteristics of the analysed subjects are presented in Table 1 .

All subjects were in good physical and mental health, based on physical examination, medical and surgical history.

\subsection{Pharmacokinetics}

The mean \pm standard deviation (SD) plasma concentration-time profiles obtained after sildenafil administration by the sublingual and supralingual routes are shown in
Table 1 Demographic data of study subjects $(N=12)$

\begin{tabular}{ll}
\hline Parameter & Value \\
\hline Sex, $[\mathrm{n}(\%)]$ & $12(100.0 \%)$ \\
$\quad$ Male & \\
Race, $[\mathrm{n}(\%)]$ & $1(8.3 \%)$ \\
Black & $11(91.7 \%)$ \\
White & \\
Age (years) & $36.2 \pm 5.2$ \\
Mean $\pm \mathrm{SD}$ & $23-45$ \\
Range & \\
Height $(\mathrm{cm})$ & $176.9 \pm 5.7$ \\
Mean $\pm \mathrm{SD}$ & $167-187$ \\
Range & \\
Body weight $(\mathrm{kg})$ & $79.5 \pm 7.1$ \\
Mean $\pm \mathrm{SD}$ & $68.5-88.4$ \\
Range & \\
Body mass index $\left(\mathrm{kg} / \mathrm{m}^{2}\right)$ & $25.5 \pm 2.9$ \\
Mean $\pm \mathrm{SD}$ & $19.6-29.3$ \\
Range &
\end{tabular}

$S D$ standard deviation

Fig. 1 for sildenafil and in Fig. 2 for the $N$-desmethylsildenafil metabolite.

The main plasma pharmacokinetic parameters data (mean $\pm \mathrm{SD}$ ) and the results of their statistical comparisons are presented in Table 2 for sildenafil and in Table 3 for $N$-desmethyl-sildenafil.

Maximum plasma concentration $\left(C_{\max }\right)$ of sildenafil was $296.50 \pm 142.69 \mathrm{ng} / \mathrm{mL}$ for the sublingual route (test) and $288.92 \pm 118.78 \mathrm{ng} / \mathrm{mL}$ for the supralingual route (reference). The peak concentration was rapidly reached, at a similar median time for the two study treatments (i.e. 0.63 and $0.75 \mathrm{~h}$ for the test and reference treatments, respectively). Both the terminal volume of distribution and the total clearance (see Table 2) were almost the same for the two administration routes. Plasma concentrations declined rapidly after $C_{\max }$ with nearly identical half-lives, i.e. 2.55 $\pm 0.26 \mathrm{~h}$ and $2.69 \pm 0.32 \mathrm{~h}$ for the sublingual and supralingual administration routes, respectively. $\mathrm{AUC}_{0-\mathrm{t}}$ values were $761.72 \pm 217.71 \mathrm{~h} \bullet \mathrm{ng} / \mathrm{mL}$ for the test treatment and 750.76 $\pm 246.10 \mathrm{~h} \bullet \mathrm{ng} / \mathrm{mL}$ for the reference. $\mathrm{AUC}_{0-\infty}$ too was very similar for the two treatments, corresponding to $798.78 \pm$ 227.68 and $793.44 \pm 269.63 \mathrm{~h} \bullet \mathrm{ng} / \mathrm{mL}$ for the sublingual and supralingual administration routes, respectively. The ratios of test/reference geometric means (point estimate, $\mathrm{PE}$ $\%)$ were $100.18,102.44$ and $102.13 \%$ for sildenafil $C_{\max }$, $\mathrm{AUC}_{0-\mathrm{t}}$ and $\mathrm{AUC}_{0-\infty}$, respectively, with their corresponding $90 \%$ CIs within the pre-specified acceptance limits for $\mathrm{AUC}_{0-\mathrm{t}}(94.90-110.58 \%)$ and $\mathrm{AUC}_{0-\infty}(94.58-110.29 \%)$ 
Fig. 1 Mean (+ SD) plasma sildenafil concentration $(\mathrm{ng} / \mathrm{mL})$ versus time profiles after single administration of sildenafil 50 $\mathrm{mg}$ orodispersible film by sublingual and supralingual route. Linear scale $N=12$
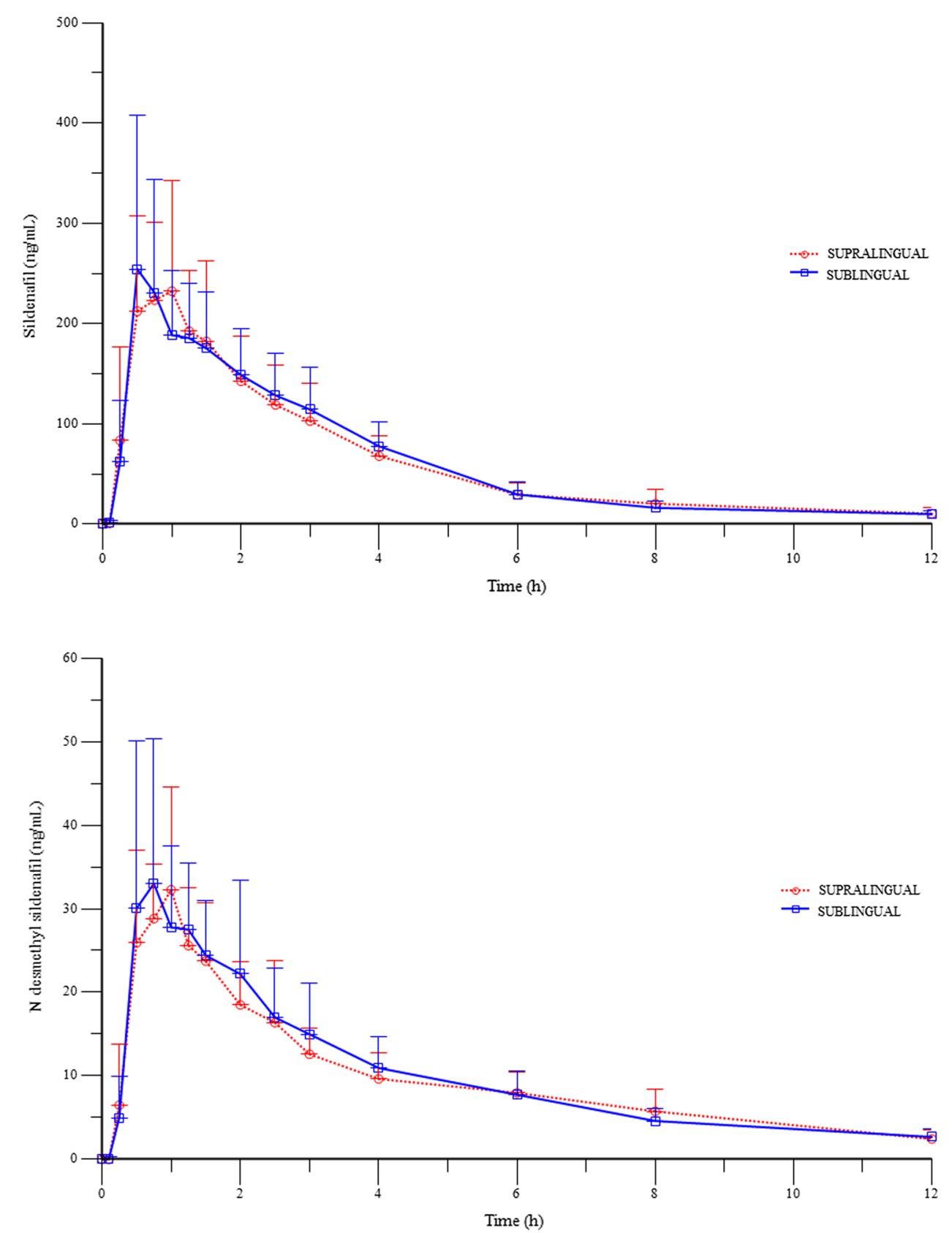

Fig. 2 Mean (+ SD) plasma $\mathrm{N}$-desmethyl-sildenafil concentration $(\mathrm{ng} / \mathrm{mL})$ versus time profiles after single administration of sildenafil $50 \mathrm{mg}$ orodispersible film by sublingual and supralingual route. Linear scale $N=12$ and only just slightly outside the $80-125 \%$ range for $C_{\max }$ (79.92-125.57\%).

$\mathrm{N}$-desmethyl-sildenafil metabolite $\mathrm{AUC}_{0-\mathrm{t}}$ and $\mathrm{AUC}_{0-\infty}$ values were, on average, similar following administration of the two treatments (see Table 3) and their 90\% CI fell within the limits of $80.00-125.00 \%$. Extent of $N$-desmethyl-sildenafil exposure was therefore equivalent when the investigational product was administered sublingually and supralingually. After sublingual administration, $N$-desmethyl-sildenafil metabolite $C_{\max }$ was approximately $10 \%$ higher compared to the supralingual route (PE \%: 109.75\%). On the other hand, the metabolite $t_{\max }$ and $t_{1 / 2}$ were nearly identical for the two administration routes.

\subsection{Safety}

Both tested treatments showed a good safety profile and no serious AEs were reported. Four subjects (33.3\%) reported mild-to-moderate headache and one subject (8.3\%) experienced vomiting during the study. These AEs were deemed by the Investigator as possibly related to study treatments. No clinically relevant effects on vital signs, ECGs or laboratory parameters were observed. 
Table 2 Sildenafil pharmacokinetic parameters and statistical analysis results after single administration of sildenafil $50 \mathrm{mg}$ orodispersible film by sublingual and supralingual route $N=12$

\begin{tabular}{lllll}
\hline Sildenafil & & & \\
\hline Pharmacokinetic parameter & Sublingual route (test) & $\begin{array}{l}\text { Supralingual route (refer- } \\
\text { ence) }\end{array}$ & PE \% & $90 \%$ CI $(\%)$ \\
\hline$C_{\max }(\mathrm{ng} / \mathrm{mL})$, mean $\pm \mathrm{SD}$ & $296.50 \pm 142.69$ & $288.92 \pm 118.78$ & 100.18 & $79.92-125.57$ \\
$t_{\max }(\mathrm{h})^{\mathrm{a}}$, median $($ range $)$ & $0.63(0.50-2.00)$ & $0.75(0.25-1.50)$ & 102.44 \\
$\mathrm{AUC}_{0-\mathrm{t}}(\mathrm{h} \bullet \mathrm{ng} / \mathrm{mL})$, mean $\pm \mathrm{SD}$ & $761.72 \pm 217.71$ & $750.76 \pm 246.10$ & 102.13 \\
$\mathrm{AUC}_{0-\infty}(\mathrm{h} \bullet \mathrm{ng} / \mathrm{mL})$, mean $\pm \mathrm{SD}$ & $798.78 \pm 227.68$ & $793.44 \pm 269.63$ & $94.90-110.58$ \\
$V_{z} / F(\mathrm{~L})$, mean $\pm \mathrm{SD}$ & $255.35 \pm 107.88$ & $266.46 \pm 75.55$ & \\
$\mathrm{Cl} / F(\mathrm{~L} / \mathrm{h})$, mean $\pm \mathrm{SD}$ & $68.50 \pm 23.90$ & $70.87 \pm 27.00$ & \\
$t_{1 / 2}(\mathrm{~h})$, mean $\pm \mathrm{SD}$ & $2.55 \pm 0.26$ & $2.69 \pm 0.32$ & \\
\hline
\end{tabular}

$A U C_{0-t}$ area under the concentration-time curve from time 0 to the last observed concentration time t, $C I$ confidence interval, $C l / F$ total clearance after oral administration, $C_{\max }$ maximum plasma concentration, $P E$ ratio test/reference of geometric means, $S D$ standard deviation, $t_{1 / 2}$ terminal half-life, $t_{\max }$ time to achieve maximum plasma concentration, $V_{z} / F$ terminal volume of distribution after oral administration

${ }^{a}$ Comparison between sublingual and supralingual route by Wilcoxon signed-rank test: $p=0.9277$

Table $3 \mathrm{~N}$-desmethyl-sildenafil pharmacokinetic parameters and statistical analysis results after single administration of sildenafil 50 mg orodispersible film by sublingual and supralingual route $N=12$

\begin{tabular}{|c|c|c|c|c|}
\hline \multicolumn{5}{|l|}{$N$-desmethyl-sildenafil } \\
\hline Pharmacokinetic parameter & Sublingual route (test) & $\begin{array}{l}\text { Supralingual route (refer- } \\
\text { ence) }\end{array}$ & $\mathrm{PE} \%$ & $90 \% \mathrm{CI}(\%)$ \\
\hline$C_{\max }(\mathrm{ng} / \mathrm{mL})$, mean $\pm \mathrm{SD}$ & $42.58 \pm 17.20$ & $37.38 \pm 11.15$ & 109.75 & $92.73-129.90$ \\
\hline$t_{\max }(\mathrm{h})^{\mathrm{a}}$, median (range) & $1.13(0.50-2.00)$ & $1.00(0.50-2.50)$ & & \\
\hline $\mathrm{AUC}_{0-t}(\mathrm{~h} \bullet \mathrm{ng} / \mathrm{mL})$, mean $\pm \mathrm{SD}$ & $121.58 \pm 33.17$ & $117.69 \pm 25.68$ & 102.09 & $91.21-114.28$ \\
\hline $\mathrm{AUC}_{0-\infty}(\mathrm{h} \bullet \mathrm{ng} / \mathrm{mL})$, mean $\pm \mathrm{SD}$ & $138.51 \pm 38.22$ & $133.73 \pm 35.94$ & 103.43 & $93.35-114.59$ \\
\hline$t_{1 / 2}(\mathrm{~h})$, mean $\pm \mathrm{SD}$ & $4.10 \pm 1.37$ & $4.07 \pm 1.28$ & & \\
\hline
\end{tabular}

$A U C_{0-t}$ area under the concentration-time curve from time 0 to the last observed concentration time t, $C I$ confidence interval, $C_{m a x}$ maximum plasma concentration, $P E$ ratio test/reference of geometric means, $S D$ standard deviation, $t_{1 / 2}$ terminal half-life, $t_{\max }$ time to achieve maximum plasma concentration

${ }^{\mathrm{a} C}$ Comparison between sublingual and supralingual route Wilcoxon signed-rank test: $p=1.0000$

\section{Discussion}

A sildenafil ODF containing sildenafil citrate has recently been marketed to meet the rising interest for sildenafil formulations able to dissolve very rapidly in the oral cavity without drinking or chewing, and to provide a more practical and user-friendly alternative to the marketed products for the treatment of ED.

Mean sildenafil plasma concentration-time profiles up to $12 \mathrm{~h}$ after single-dose sublingual and supralingual administration were nearly superimposable. The ratio of test/reference geometric means was very close to $100 \%$ for sildenafil $C_{\max }, \mathrm{AUC}_{0-\mathrm{t}}$ and $\mathrm{AUC}_{0-\infty}$, indicating a comparable rate and extent of sildenafil exposure with the two treatments. The $90 \%$ CIs were within the equivalence limits of $80.00-125.00 \%$ for the two AUCs and just slightly outside the pre-specified limits for the peak concentration, due to a high within-subject variability and the limited number of subjects in the study. The nearly identical $t_{\max }$, volume of distribution, total clearance and $t_{1 / 2}$ values obtained with the two administration routes confirmed that sildenafil presented the same absorption, distribution and elimination kinetics after sublingual and supralingual dosing.

A comparison of the present results with the literature data [13], obtained after supralingual administration of the sildenafil $100 \mathrm{mg}$ ODF during a bioequivalence study versus Viagra ${ }^{\circledR} 100 \mathrm{mg}$, showed similar rate and extent of exposure.

The data obtained in the present study and previously in the bioequivalence study between the ODF formulation and Viagra ${ }^{\circledR}$ film-coated tablets [13] indicated that the absorption of sildenafil in the mucosa of the oral cavity was negligible and had no significant impact on the drug kinetics. 
After the complete dissolution of the ODF in the mouth, sildenafil was likely swallowed and absorbed further down in the gastro-intestinal tract, regardless of the place of disaggregation (sublingual or supralingual) of the film.

The $90 \%$ CIs for $N$-desmethyl sildenafil AUCs fell within the limits of $80.00-125.00 \%$, and therefore the two administration routes were also equivalent with respect to the extent of metabolite exposure. The peak metabolite plasma concentration was slightly different between the two treatments, likely due to the small sample size considered. The sublingual route of administration showed a $10 \%$ higher $C_{\max }$ and, consequently, the $90 \% \mathrm{CI}$ did not fall within the acceptance limits of $80.00-125.00 \%$. However, the metabolite $t_{\max }$ and $t_{1 / 2}$ values were nearly identical $(p=1.0000)$ for the two administration methods, confirming that both the formation rate and the elimination rate of the metabolite were not significantly different. Considering that $N$-desmethyl sildenafil had a peak concentration less than $15 \%$ of its parent compound and $40 \%$ of its biological activity, the difference between the two administration routes in the metabolite $C_{\max }$ value can be considered clinically not significant.

Safety data confirmed a favourable safety profile of the investigational product, administered as single oral dose of $50 \mathrm{mg}$ sildenafil by both the sublingual and supralingual routes.

The limited sample size (due to the exploratory nature of the study) did not permit to establish a formal bioequivalence between the two routes of administration, but the design and number of subject enrolled is deemed adequate by international guidelines [14] to obtain a reliable pharmacokinetic and statistical comparison between treatments.

\section{Conclusion}

In conclusion, in healthy men the sublingual administration of sildenafil $50 \mathrm{mg}$ ODF produced the same pharmacokinetic profile as the supralingual administration. Study data confirm the similarity between the two routes of administration, thus suggesting that sildenafil ODF formulation, administered both sublingually and supralingually, can be an effective and safe treatment for ED.

Acknowledgements We would like to gratefully acknowledge CROSS Research S.A. for study coordination, Analytisch Biochemisch Laboratorium BV (The Netherlands) for sildenafil and $N$-desmethyl-sildenafil bio-analyses.

\section{Complaince with Ethical Standards}

Funding This study was funded by IBSA Institut Biochimique SA., Switzerland.

Conflict of interest V.F. is an employee of IBSA Institut Biochimique SA, L.L., C.L. and M.R. are employees of CROSS Research S.A., which was contracted by IBSA Institut Biochimique SA as CRO for the conduction of this study and received financial support for its services. The authors declare that they have no other relationships or activities that could appear to have influenced the submitted work.

Ethical approval The study protocol (code 17CH-SDF06) was approved by the Ethics Committee of Canton Ticino, Switzerland, and the Swiss Federal Health Authorities.

Informed consent All subjects provided written informed consent before enrolment.

Open Access This article is distributed under the terms of the Creative Commons Attribution-NonCommercial 4.0 International License (http://creativecommons.org/licenses/by-nc/4.0/), which permits any noncommercial use, distribution, and reproduction in any medium, provided you give appropriate credit to the original author(s) and the source, provide a link to the Creative Commons license, and indicate if changes were made.

\section{References}

1. Yafi FA, Jenkins L, Albersen M, Corona G, Isidori AM, Goldfarb S, et al. Erectile dysfunction. Nat Rev Dis Primers. 2016;2:16003.

2. Andersson KE. Mechanisms of penile erection and basis for pharmacological treatment of erectile dysfunction. Pharmacol Rev. 2011;63(4):811-59.

3. Gratzke C, Angulo J, Chitaley K, Dai YT, Kim NN, Paick JS, et al. Anatomy, physiology, and pathophysiology of erectile dysfunction. J Sex Med. 2010;7(1 Pt 2):445-75.

4. Bender AT, Beavo JA. Cyclic nucleotide phosphodiesterases: molecular regulation to clinical use. Pharmacol Rev. 2006;58(3):488-520.

5. Puzzo D, Sapienza S, Arancio O, Palmeri A. Role of phosphodiesterase 5 in synaptic plasticity and memory. Neuropsychiatr Dis Treat. 2008;4(2):371-87.

6. Goldstein I, Lue TF, Padma-Nathan H, Rosen RC, Steers WD, Wicker PA. Oral sildenafil in the treatment of erectile dysfunction. Sildenafil Study Group. N Engl J Med. 1998;338(20):1397-404.

7. Nichols DJ, Muirhead GJ, Harness JA. Pharmacokinetics of sildenafil after single oral doses in healthy male subjects: absolute bioavailability, food effects and dose proportionality. Br J Clin Pharmacol. 2002;53(Suppl 1):5S-12S.

8. Moncada I, Jara J, Subira D, Castano I, Hernandez C. Efficacy of sildenafil citrate at 12 hours after dosing: re-exploring the therapeutic window. Eur Urol. 2004;46(3):357-60 (discussion 360-1).

9. Hyland R, Roe EG, Jones BC, Smith DA. Identification of the cytochrome $\mathrm{P} 450$ enzymes involved in the $\mathrm{N}$-demethylation of sildenafil. Br J Clin Pharmacol. 2001;51(3):239-48.

10. Muirhead GJ, Rance DJ, Walker DK, Wastall P. Comparative human pharmacokinetics and metabolism of single-dose oral and intravenous sildenafil. Br J Clin Pharmacol. 2002;53(Suppl 1): $13 \mathrm{~S}-20 \mathrm{~S}$

11. Walker DK, Ackland MJ, James GC, Muirhead GJ, Rance DJ, Wastall P, et al. Pharmacokinetics and metabolism of sildenafil in mouse, rat, rabbit, dog and man. Xenobiotica. 1999;29(3):297-310.

12. Viagra ${ }^{\circledR}$ (sildenafil citrate) tablets, for oral use, full prescribing information. LAB-0220-11.0, Pfizer Labs, Revised: 08/2017.

13. Radicioni M, Castiglioni C, Giori A, Cupone I, Frangione V, Rovati S. Bioequivalence study of a new sildenafil $100 \mathrm{mg}$ orodispersible film compared to the conventional film-coated 100 
mg tablet administered to healthy male volunteers. Drug Des Dev Ther. 2017;11:1183-92.

14. Guidance on the investigation of bioequivalence. CPMP/EWP/ QWP/1401/98 Rev. 1/Corr **, London, 20 Jan 2010. European Medicines Agency, Committee for Medicinal Products for Human Use.

15. Gerrits M, de Greef R, Peeters P. Effect of absorption site on the pharmacokinetics of sublingual asenapine in healthy male subjects. Biopharm Drug Dispos. 2010;31(5-6):351-7.
16. Guideline on bioanalytical method validation. EMEA/CHMP/ EWP/192217/2009 Rev. 1 Corr.*, 21 Jul 2011. European Medicines Agency, Committee for Medicinal Products for Human Use.

17. Guidance for Industry. Bioanalytical method validation. New York: U.S. Department of Health and Human Services Food and Drug Administration; 2001. 